\title{
Generation of Plasma Rotation in a Tokamak by Ion-Cyclotron Absorption of Fast Alfven Waves
}

\author{
F. W. Perkins ${ }^{1,2}$, R. B. White ${ }^{2}$, and P. Bonoli ${ }^{3}$ \\ ${ }^{1}$ Plasma Physics Laboratory, P.O.Box 451, Princeton, New Jersey 08543 \\ ${ }^{2}$ General Atomics, PO Box 85608, San Diego, CA, 92186-5608, USA \\ ${ }^{3}$ Plasma Science and Fusion Center, MIT, Cambridge, MA 02139-4307, USA
}

Control of rotation in tokamak plasmas provides a method for suppressing fine-scale turbulent transport by velocity shear and for stabilizing large-scale magnetohydrodynamic instabilites via a close-fitting conducting shell. The experimental discovery of rotation in a plasma heated by the fast-wave minority ion cyclotron process is important both as a potential control method for a fusion reactor and as a fundamental issue, because rotation arises even though this heating process introduces negligible angular momentum [1-4]

This paper proposes and evaluates a mechanism which resolves this apparent conflict. First, it is assumed that angular momentum transport in a tokamak is governed by a diffusion equation with a no-slip boundary condition at the plasma surface and with a torque-density source that is a function of radius. When the torque density source consists of two separated regions of positive and negative torque density, a non-zero central rotation velocity results, even when the total angular momentum input vanishes. Secondly, we show that localized ion-cyclotron heating can generate regions of positive and negative torque density and consequently central plasma rotation.

Recent papers by $V$. Chan [5] and K. Ida [6] review plasma rotation induced by radio frequency power and radial electric fields. Alcator C-Mod observations have established that the central rotation velocity increases roughly linearly with the plasma energy content and that rotation is strongly peaked toward the plasma center when the ion-cyclotron resonance is close to the magnetic axis $[1,2]$. Co-current rotation reported in ohmicallyheated H-mode plasmas [7] lies outside the scope of this paper, but could possibily be understood in terms of a modification of our no-slip boundary condition. This work differs from previous theoretical models[8] in its rigorous accounting of angular momentum, the role of radial currents associated with energetic-ion banana diffusion, and the use of a diffusive transport equation to describe plasma response to torques.

We separate the plasma into two components: a high-energy tail created by minority ion-cyclotron heating and a bulk plasma, which responds to applied torque density via a diffusive angular momentum transport equation with an empirical momentum diffusivity profile $\chi=a^{2} q^{n} / C_{n} \tau_{M}$, where $\tau_{M}$ is a momentum confinement time taken to be comparable to the energy confinement time $\tau_{E}[6]$. We report results for a uniform diffusivity, $\mathrm{n}=0, C_{n}=6$. Our model for ion-cyclotron heating is instantaneous energization of bulk plasma ions, constrained to introduce zero net angular momentum. The energetic ions are then followed as they lose energy and redistribute themselves over a region given by the diffusion in one slowing down time by the Monte-Carlo ORBIT code $[9,10]$, which includes ion-ion pitch-angle-scattering and drag collisions [11]. The particle displacements, which are both outward and inward, and can include loss, constitute radial currents. Any radial current in the energetic tail particles must be compensated by an equal but opposite radial current in the bulk plasma which creates a $R j_{r} B_{\theta}$ torque density. Collisions between 


\section{DISCLAIMER}

This report was prepared as an account of work sponsored by an agency of the United States Government. Neither the United States Government nor any agency thereof, nor any of their employees, make any warranty, express or implied, or assumes any legal liability or responsibility for the accuracy, completeness, or usefulness of any information, apparatus, product, or process disciosed, or represents that its use would not infringe privately owned rights. Reference herein to any specific commercial product, process, or service by trade name, trademark, manufacturer, or otherwise does not necessarily constitute or imply its endorsement, recommendation, or favoring by the United States Government or any agency thereof. The views and opinions of authors expressed herein do not necessarily state or reflect those of the United States Government or any agency thereof. 


\section{DISCLAIMER}

Portions of this document may be illegible in electronic image products. Images are produced from the best available original document. 

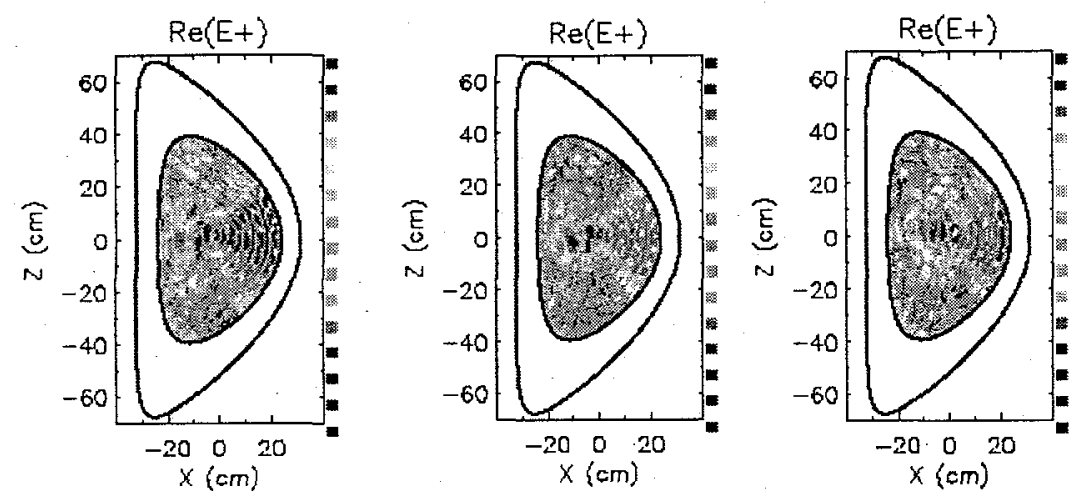

Figure 1: ICRF deposition intensity for resonance left of axis, on axis, and right of axis

energetic particles and the bulk plasma can also transfer angular momentum from the energetic particles to the bulk plasma creating a second source of torque density.

The neutralizing radial current in the bulk plasma generates a co-current torque inside the original surface and a counter-current torque outside. Such a torque distribution will lead to a nonzero, co-current central rotation. However, because torque associated with collisional angular momentum transfer is comparable to the $R j_{r} B_{\theta}$ torque, a numerical solution that rigorously accounts for all angular momentum is needed. Bulk plasma response to an applied torque is taken to be governed by a flux-surface-average steadystate angular momentum diffusion equation

$$
\frac{1}{V^{\prime}} \frac{\partial}{\partial \psi}\left(\left\langle n M R^{2} \chi(\nabla \psi)^{2}\right\rangle V^{\prime} \frac{\partial \Omega}{\partial \psi}\right)=-\langle\tau\rangle
$$

written in nondimensional units, with the fundamental mass, length and frequency units being the deuteron mass, the major radius $R$, and the on-axis cyclotron frequency $\omega_{c i}$ respectively. Here $\Omega$ denotes the bulk plasma rotation frequency, $V^{\prime}=\partial_{\psi} V$, and $\langle>$ is a flux-surface average. The nondimensional toroidal flux $\Phi$ is taken to be the fundamental independent variable and flux surface label with $d \Phi / q=d \psi$. The solution to Eq. 1 with a no-slip boundary condition at the plasma surface, arising from the observation that the separatrix flux surface is line-tied to a fixed conducting material boundary, is

$$
\Omega(\Phi)=\int_{\Phi}^{\Phi_{\max }} \frac{d \Phi^{\prime} T\left(\Phi^{\prime}\right)}{q V^{\prime}\left\langle n M R^{2} \chi(\nabla \psi)^{2}\right\rangle}
$$

where $T=\int_{0}^{\Phi} d \Phi^{\prime}\langle\tau\rangle V^{\prime} / q$ is the nondimensional integrated torque applied inside flux surface $\Phi$. The integrated torque will also have a surface contribution when particles are lost from the plasma. Zero angular momentum input is $T\left(\Phi_{\max }\right)=0$.

In a circular flux surface tokamak these expressions are: $V^{\prime}=(2 \pi)^{2} q, r^{2}=2 \Phi, n=$ $n_{e} R^{3}, \chi=\chi_{0} q^{n}=a^{2} q^{n}\left(C_{n} R^{2} \omega_{c i} \tau_{M}\right)^{-1}$, and $q=1+3\left(\Phi / \Phi_{\max }\right)$. Since the principal contribution to the integral for $\Omega$ is expected to come from a thin layer whose thickness scales with the gyroradius, this integral will be rescaled by a factor $v^{-1}$, where $v=$ $(2 E / M)^{1 / 2}\left(R \omega_{c i}\right)^{-1}$. We also introduce $T^{*}$, the angular momentum transferred from an average energetic particle to the bulk plasma inside flux surface $\Phi$, via $T=\dot{N} T^{*} v$ where $\dot{N}$ is the rate at which the plasma heating is supplying energetic particles of energy $\mathrm{E}$, so 
the fast-wave heating power $P=\dot{N} E$, giving

$$
\frac{\Omega(\Phi)}{\dot{N}}=v \int_{\Phi}^{\Phi_{\max }} \frac{d \Phi^{\prime} T^{*}\left(\Phi^{\prime}\right)}{2(2 \pi)^{2}\langle n \chi\rangle} \equiv \frac{v^{2}}{2(2 \pi)^{2}\left\langle n \chi_{0}\right\rangle} I_{n}^{*}(\Phi)
$$

With this scaling, $I^{*}$ is insensitive to particle energy.

The initial conditions for ORBIT constitute our model for ion cyclotron heating, wherin particles are instantly energized to a given energy $E$, their banana tips are placed at the cyclotron resonance surface $R=R_{c}$ and are distributed in $z$ according to $d N / d z=$ $\left(z_{m}^{2}-z^{2}\right) /\left(z_{m}^{2}\left(2 z_{m}^{2}-z^{2}\right)^{1 / 2}\right)$. Our choice of $z_{m}$ is guided by TORIC wave propagation and absorption calculations. Fig. 1 shows the wave fields for the resonant surfaces. Regions of intense fields lie close to the equatorial plane and motivate our choice $z_{m}=6.7 \mathrm{~cm}$.

The collisions are given by $d\left\langle\theta^{2}\right\rangle / d t=\nu_{0}\left(E_{0} / E\right)^{3 / 2}, d E / E d t=-\nu_{0}\left(E_{0} / E\right)^{3 / 2}\left(M_{p} / M_{d}\right)$, where $\nu_{0}$ is the collision frequency and $E_{0}$ is the initial particle energy. As long as the transport is in the banana regime the orbit evolution is insensitive to $\nu_{0}$, but the ratio of pitch-angle scattering to energy drag is important. ORBIT records the angular momentum increment $M R\left(\Delta v_{\|}\right)$for each collision event in one of the 50,000 computational bins in toroidal flux corresponding to the magnetic surface where the collision occured. From this data one forms $T_{2}^{*}=\int_{0}^{\Phi} M R \Delta v_{\|} d \Phi$, which is the ensemble average angular momentum impulse imparted to the bulk plasma within flux surface $\Phi$ by collisions.

Torque also arises from the radial currents which result when a particle comes to rest on a magnetic surface which differs from their original one. The total torque $\delta T$ exerted on a shell of thickness $\delta \psi$ in poloidal flux is given by the radial current $I_{r}$, determined in turn by the fraction of particles which come to rest inside a given magnetic surface. For each Monte Carlo particle, we record the initial magnetic flux surface $\Phi_{0}$ and its final position is assigned to one of the bins. From this data one can form $T_{1}^{*}(\Phi)=(1 / v) \int_{0}^{\Phi} d \Phi^{\prime} G\left(\Phi^{\prime}\right) / q$, $G(\Phi)=F(\Phi)$ for $\Phi<\Phi_{0}$ and $F(\Phi)-1$ for $\Phi>\Phi_{0}$, and $F(\Phi)$ is the the average number of particles whose final position is inside surface $\Phi . T_{1}^{*}$ is the angular momentum given to the bulk plasma by a single ensemble-average particle through $R j_{r} B_{\theta}$ torques. Monte Carlo runs determine $F(\Phi), T_{1}^{*}(\Phi), T_{2}^{*}(\Phi)$, and finally $I^{*}(\Phi)$. Because our final expression involves two integrations over the distributions, the results a very insensitive to bin statistics and adequate accuracy results from 500 Monte Carlo particles per run.

To compare our results with experiment, let us recast Eq.(3) into dimensional form and equate $\dot{N} E=P_{a u x}$ and $P_{a u x} \tau_{E}=W$. Figure 2 a presents the nondimensional rotation profile $I^{*}(\Phi)$ for a scan of resonance surface locations. In the case of Alcator C-Mod, representative parameters are $E=48 \mathrm{keV}, B=5.3 T, W=150 \mathrm{~kJ}, n_{\epsilon}=2 \times 10^{20} \mathrm{~m}^{-3}$, $R=.67 \mathrm{~m}, R_{c}=.71 \mathrm{~m}, a=0.22 \mathrm{~m}$, and $I^{*}(0)=8.8$. For a spatially uniform momentum diffusivity $\mathrm{n}=0$ and $C_{n}=6$. The expression for on-axis rotational frequency is then

$$
\Omega=\left[\frac{C_{n} W}{e B R^{3} a^{2} \bar{n}(2 \pi)^{2}}\left(\frac{\tau_{m}}{\tau_{E}}\right)\right] I_{n}^{*}
$$

For the parameters above, $\Omega=8.2 \times 10^{4} \mathrm{rad} / \mathrm{s}$, which lies in the range of observed results, is in the co-current direction, and has the centrally peaked velocity profile characteristic of experiments. Particle energy E no longer enters Eq. 4 explicitly, having been incorporated into the auxiliary heating power and then into the plasma energy content. $I_{n}^{*}(\Phi)$ is a 

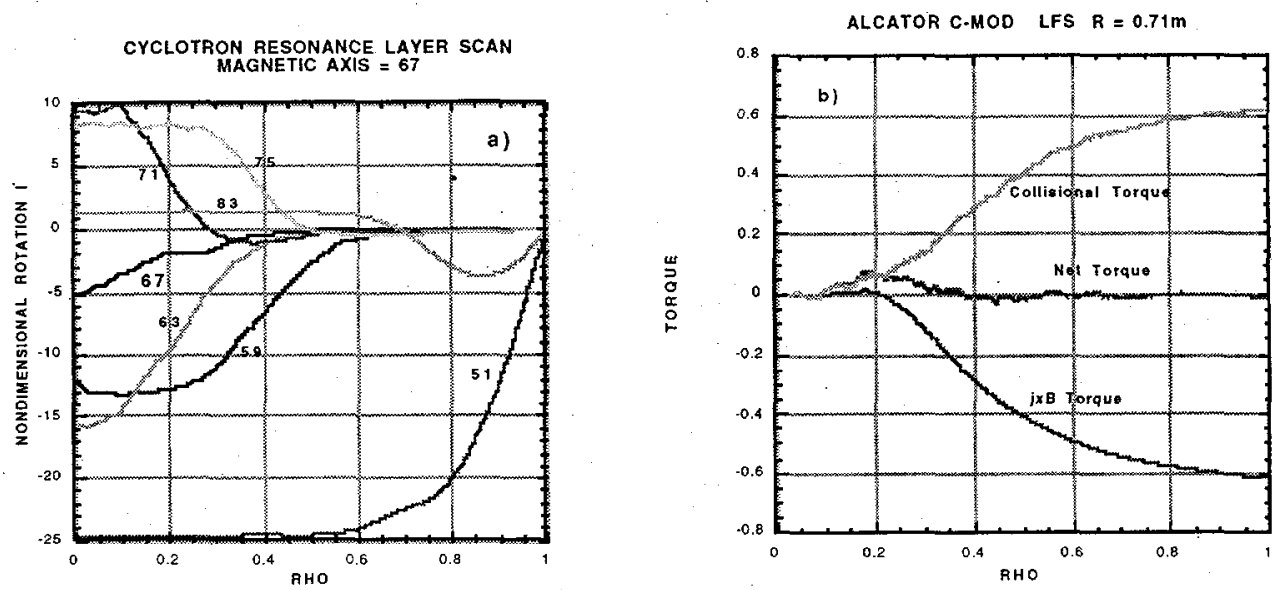

Figure 2: Rotation profiles for different resonance locations (left), and torques induced by ICRF (right)

nondimensional angular rotation frequency profile. The nondimensional integrated torque profiles portrayed in Fig. $2 \mathrm{~b}$ show that the region of net integrated torque is concentrated near the cyclotron resonance layer and that the net angular momentum input vanishes. The cancellation of $R j_{r} B_{\theta}$ torque with collisional mechanical angular momentum torque in the outer region of the plasma is associated with ion banana diffusion and illustrates the need for the rigorous conservation of angular momentum which ORBIT provides.

Equation 4 is our principal result. It connects rotation rates with solutions of the nondimensional Monte Carlo computational procedure. Studies have been carried out to assess the sensitivity of our baseline results to input parameters and properties of the ioncyclotron heating model. The on-axis nondimensional rotation $I^{*}(0)$ increases by about a factor of 2 for an ICRF model which starts particles in pairs on the equatorial plane with equal but opposite parallel velocity. There is little variation of $I^{*}$ with initial particle energy or pitch, i.e. Eq. 4 has the correct scaling. For high field side resonance locations, counter-current rotation is found for both the baseline "banana tips" ICRF model and the equatorial plane model. Overall, we conclude that ion-cyclotron particle energization provides a theoretical basis for understanding driven plasma rotation on magnetic surfaces inside the resonance surface. Improvements in the ion cyclotron heating model, boundary condition, and diffusivity profiles should increase correspondence with observatiions.

This work was supported by the U.S. Department of Energy contract number DE-AC02-76-CHO3073.

[1] Rice, J. E. et. al., Nuclear Fusion 39, 1175 (1999).

[2] Rice, J. E. et. al., Nuclear Fusion 38, 75 (1998).

[3] Hoang, G. T. et. al., in Radio Frequency Power in Plasmas 1999 (American Institute of Physics) 132.

[4] Eriksson, L.-G. et. al., Plasma Phys. Control. Fusion 39, 27 (1997).

[5] Chan, V. et. al., in Radio Frequency Power in Plasmas 1999 (American Institute of Physics) 45.

[6] Ida, K. et. al., Plasma Phys. Control. Fusion 40, 1429 (1998).

[7] Hutchinson, I. H. et. al., Phys. Rev. Lett. 84, 3330 (2000).

[8] Chang, C. S. et. al., Phys. Plasmas 6, 1969 (1999).

[9] White, R. B., and M. S. Chance, Phys. Fluids 27, 2455 (1984).

[10] White, R. B. Phys. Fluids B 2, 845 (1990).

[11] Boozer, A. H., and G. Kuo-Petravic Phys. Fluids 25, 851 (1981). 